\title{
Cytokines and anti-cytokine therapy in periodontal disease
}

\author{
Agraja Patil ${ }^{1}$, Swapna Mahale ${ }^{2}$ \\ ${ }^{1}$ (Post- graduate student, Department of Periodontology, MGV KBH Dental College, Nashik) \\ ${ }_{2}^{2}$ (Professor, Guide and HOD, Department of Periodontology, MGV KBH Dental College, Nashik)
}

\begin{abstract}
Periodontal disese is initiated by the presence of bacteria on tooth surface but it is the host response that helps in progression of disease. An intermediate mechanism that lies between bacterial stimulation and tissue destruction is the production of cytokines, which stimulates inflammatory events that activate effector mechanisms. There are various classes of cytokines depending on their chemical structure and origin. Owing to the unique mechanism of action, these micromolecules play a very important part in immune host defence. Anticytokine therapy for periodontal diseases especially targets proinflammatory cytokines, that is, TNF- $\alpha, I L-1$, and IL-6, because these are essential for the initiation of the inflammatory immune reaction and are produced for prolonged periods in periodontitis. This therapy aims to bind the cytokines with the receptors present on target cells such as the fibroblasts.
\end{abstract}

Keywords - Chemokines, Cytokine storm, Inflammation, Interleukins, Receptors

\section{Introduction}

The immune system is a remarkably versatile defense system that has evolved to protect animals from invading pathogenic microorganisms. It is able to generate an enormous variety of cells and molecules capable of specifically recognizing and eliminating an apparently limitless variety of foreign invaders. These cells and molecules act together in a dynamic network. Effective immune response is given by Lymphoid cells, Inflammatory cells and Hematopoietic cells.

\section{Definition}

Cytokines are small secreted proteins released by cells have a specific effect on the interactions and communications between cells. ${ }^{[2]}$ They are low-molecular weight regulatory proteins or glycoproteins secreted by white blood cells and various other cells in the body in response to a number of stimuli. These proteins assist in regulating the development of immune effector cells, and some cytokines possess direct effector functions of their own.

\section{Terminologies}

Although these other two terms continue to be used, they are misleading because secretion of many lymphokines and monokines is not limited to lymphocytes and monocytes as these terms imply, but extends to a broad spectrum of cells and types. For this reason, the more inclusive term cytokine is preferred. Many cytokines are referred to as interleukins, a name indicating that they are secreted by some leukocytes and act upon other leukocytes. (Inter- within, leukins- derived from leukocytes). Some cytokines are known by common names, including the interferons and tumor necrosis factors. Recently gaining prominence is yet another another subgroup of cytokines, the chemokines a group of low-molecular weight cytokines affect chemotaxis and other aspects of leukocyte behavior. ${ }^{[3]}$

\section{Mechanism Of Action}

Cytokines bind to specific receptors on the membrane of target cells, triggering signal-transduction pathways that ultimately alter gene expression in the target cells. The susceptibility of the target cell to a particular cytokine is determined by the presence of specific membrane receptors. ${ }^{[4]}$ In general, the cytokines and their receptors exhibit very high affinity for each other, with dissociation constants ranging from $10^{-10}$ to 10

${ }^{12} \mathrm{M}$. Because their affinities are so high, cytokines can mediate biological effects at picomolar concentrations.

\subsection{Are They Hormones?}

Hormones: Generally act long range in an endocrine fashion, Are produced by specialized glands and tend to have a unique action on one or a few types of target cell.

Cytokines: Act over a short distance in an autocrine or paracrine fashion. Cytokines are often produced by, and bind to, a variety of cells. Cytokines regulate the intensity and duration of the immune response by stimulating or inhibiting the activation, proliferation, and/ or differentiation of various cells and by regulating the secretion of antibodies or other cytokines. Binding of a given cytokine to responsive target cells generally stimulates increased expression of cytokine receptors and secretion of other cytokines, which affect other target cells in 
turn. Thus, the cytokines secreted by even a small number of lymphocytes activated by antigen can influence the activity of numerous cells involved in the immune response.

\section{Properties Of Cytokines}

1. Pleiotrophy: A given cytokine that has different biological effects on different target cells has a pleiotropic action.

2. Redundancy: Two or more cytokines that mediate similar functions are said to be redundant. Redundancy makes it difficult to ascribe a particular activity to a single cytokine.

3. Synergy: Cytokine synergism occurs when the combined effect of two cytokines on cellular activity is greater than the additive effects of the individual cytokines.

4. Antagonism: Cytokines exhibit antagonism; that is, the effects of one cytokine inhibit or offset the effects of another cytokine.

5. Cascade induction: Cascade induction occurs when the action of one cytokine on a target cell induces that cell to produce one or more other cytokines, which in turn may induce other target cells to produce other cytokines.

\subsection{Biological Functions}

- Development of cellular and humoral immune responses, act in an antigen-nonspecific manner.

- They affect whatever cells they encounter that bear appropriate receptors and are in a physiological state that allows them to respond.

- Induction of the inflammatory response,

- Regulation of hematopoiesis,

- Control of cellular proliferation and differentiation,

- Healing of wounds

\section{Cytokine Receptors}

To exert their biological effects, cytokines must first bind to specific receptors expressed on the membrane of responsive target cells. Because these receptors are expressed by many types of cells, the cytokines can affect a diverse array of cells. ${ }^{[6]}$

- Immunoglobulin super family receptors

- Class I cytokine receptor family (also known as the hematopoietin receptor family)

- Class II cytokine receptor family (also known as the interferon receptor family)

- TNF receptor family

- Chemokine receptor family

A common feature to most of the hematopoietin (class I cytokine) and the class II cytokine receptor families is multiple subunits, often including one subunit that binds specific cytokine molecules and another that mediates signal transduction. Several subfamilies of class I cytokine receptors have been identified, with all the receptors in a subfamily having an identical signal-transducing subunit.

- GM-CSF

- $\quad$ IL-2

- $\quad$ IL-6

\subsection{The GM-CSF receptor subfamily}

It includes the receptors for IL-3, IL-5, and GM-CSF. Each of these cytokines binds to a unique low affinity, cytokine-specific receptor consisting of a subunit only. All three low-affinity subunits can associate non-covalently with a common signal-transducing subunit. The resulting dimeric receptor not only exhibits increased affinity for the cytokine but also can transduce a signal across the membrane after binding the cytokine.

\section{Signalling Pathway}

The cytokine receptor is composed of separate subunits, A chain required for cytokine binding and for signal transduction and A chain necessary for signaling but with only a minor role in binding. Different inactive protein tyrosine kinases are associated with different subunits of the receptor. The chain of the receptor is associated with a novel family of protein tyrosine kinases, the Janus kinase (JAK) family. Cytokine binding induces the association of the two separate cytokine receptor subunits and activation of the receptor-associated JAKs. Once receptor associated JAKs are activated, they phosphorylate specific tyrosines in the receptor subunits of the complex. Members of a family of transcription factors known as STATs (signal transducers and 
activators of transcription) bind to these phosphorylated tyrosine residues. After undergoing JAK-mediated phosphorylation, STAT transcription factors translocate from receptor docking sites at the membrane to the nucleus, where they initiate the transcription of specific genes. The STAT dimers then translocate into the nucleus and induce the expression of genes containing appropriate regulatory sequences in their promoter regions.

\subsection{Based on type of immunity ${ }^{[8]}$}

\section{Classification Of Cytokines}

The immune system can be classified as acquired and innate. Cytokines asscociated with innate immunity are IL-1, Tumour necrosis factor-alpha (TNF-alpha), IL-12, IL-6, Interferon alpha (IFN-alpha), Interferon beta (IFN-beta). Cytokines associated with adaptive immunity are IL-2, IL-4, IL-5, IL-25, Transforming growth factor beta (TGF-beta) and interferon gamma (IFN-gamma).

\subsection{On the basis of structure}

- The hematopoietin family,

- The interferon family

- The chemokine family

- The tumor necrosis factor family.

7.3 Based on the mode of action

\begin{tabular}{|c|c|c|c|c|}
\hline $\begin{array}{l}\text { Pro- } \\
\text { inflammatory }\end{array}$ & $\begin{array}{l}\text { That regulate } \\
\text { lymphocyte } \\
\text { growth, } \\
\text { activation \& } \\
\text { proliferation }\end{array}$ & $\begin{array}{l}\text { That help in } \\
\text { hematopoiesis }\end{array}$ & Chemokines & $\begin{array}{l}\text { With immuno- } \\
\text { regulatory } \\
\text { functions }\end{array}$ \\
\hline $\begin{array}{ll} & \text { IL-1 } \\
\text { - } & \text { TNF alpha } \\
& \text { \& beta } \\
\text { - } & \text { IL-6 } \\
\text { - } & \text { IL-8 }\end{array}$ & $\begin{array}{ll}\bullet & \text { IL } 2 \\
\bullet & \text { IL 4 } \\
\text { - } & \text { IL 5 } \\
\text { • } & \text { IL 12 } \\
\text { - } & \text { IL 15 } \\
\text { - } & \text { TGF beta }\end{array}$ & $\begin{array}{lc}\cdot & \text { IL } 1 \\
\text { - } & \text { IL 3 } \\
\text { - } & \text { IL 6 } \\
\text { - } & \text { IL } 7\end{array}$ & $\begin{array}{ll} & \text { Alpha } \\
\text { - } & \text { Beta }\end{array}$ & $\begin{array}{ll}\text { - } & \text { Interferons } \\
\text { - } & \text { IL } 12 \\
& \text { IL } 18\end{array}$ \\
\hline
\end{tabular}

\subsubsection{Interleukin 1:}

It has 2 molecular forms-IL-1 $\alpha$,IL-1 $\beta$. Encoded by 2 separate genes. Main source of IL- 1 are the cells of monocyte-macrophage lineage. IL- $1 \alpha$ tends to remain associated to membranes. IL-1 $\beta$ - synthesised as an inactive precursor, released from the cell after being processed post- transitionally by a cysteine-asparagene protease. IL- $1 \beta$ is expressed by monocytes, macrophages and dendritic cells. It signals through 2 receptors- IL1R1 and IL-1RII both of which are shared with IL-1 ${ }^{\alpha}$. It is a multifunctional molecule the effects ranging from inflammation, immunity and hemopoiesis. IL-1 has diverse activities and roles in immunity,inflammation, tissue breakdown and tissue homeostasis.

Role in periodontitis: From human and animal models, there is strong evidence of a role for IL-1 in mediating bone loss stimulated by periodontal pathogens. Charon and co-workers were first to demonstrate that GCF of inflamed sites of gingivitis had $\uparrow$ IL-1 activity. Hendley et al(1995) suggested that neutrophils may be a source for IL- $\beta$ in periodontal disease. IL-1 $\beta$ levels are higher in gingiva of patients with active disease and is stimulator of destruction of periodontal ligament and alveolar bone, induces secretion of MMP's and prostaglandins. IL-1 mRNA was higher in connective tissue farthest from the pocket epithelium. Keratinocytes in gingival tissues have been demonstrated to produce IL-1 $\alpha$ (Matsuki et al - 1993). Transgenic mice overexpressing IL-1 $\alpha$ in gingival epithelium developed a syndrome to classic features of periodontal disease, including loss of attachment and destruction of alveolar bone. Delima et al. showed that inhibition of IL-1 $\alpha$ using human soluble IL-1 receptor type I (IL-1R) significantly reduced inflammation, connective tissue attachment loss, and bone resorption induced by periodontal pathogens compared to control.

\subsubsection{Interleukin 6}

It is produced by variety of cells like monocytes, fibroblasts, osteoblasts and vascular endothelial cells in response to inflammatory changes. Has pro-inflammatory and hematopoietic activity. Role as a proinflammatory cytokine is similar to IL-1 and TNF. Action on IL-6 on B-cells is the increased secretion of Ig-M, Induces T-cell proliferation and is a pleiotropic cytokine.

IL-6 levels are shown to increase in GCF of refractory periodontitis (Rienhardt et al - 1993). IL-6 message has been detected in inflamed gingival tissues but not in healthy tissues (Takahashi et al - 1994). IL-6 appears to have a role in bone resorption by stimulating formation of multinucleated cells similar to osteoclasts. 
Reddi et al showed that surface associated material from A.a and other oral pathogens induced IL-6 but not IL-1 or TNF from gingival fibroblasts

\subsubsection{Interleukin 8}

Chemo-attractant for neutrophils. Induced and secreted by monocytes, lymphocytes, fibroblasts, epithelial and endothelial cells. Induces the adhesion of PMN to endothelial cells and their trans-endothelial migration and release of granulocyte enzymes from these cells, promotes angiogenesis and inhibits endothelial apoptosis. IL-8 has been detected in GCF of adult periodontitis patients. IL-8 mRNA has been shown in both healthy and diseased tissues in the junctional and sulcular epithelium. LPS stimulated PMN's release IL-8 and IL-1ra which results in blocking synthesis of IL-8 in monocytes (Granowitz et al - 1992). A.a and C.rectus have been shown to stimulate IL-6 and IL-8 production from human gingival fibroblasts (Dongari et al - 1996)

\subsubsection{TNF:}

Name can be misleading, does not cause necrosis of tumours rather causes apoptosis (cell death). Two members of the family

- Tumour necrosis factor (formerly known as TNF- $\alpha$ )

- Lymphotoxin $\beta$ (formerly known as TNF- $\beta$ )

Secreted by macrophages principally-Monocytes, neutrophils, T-cells, NK cells

It is a mediator of natural and acquired immunity. Local increased concentrations cause heat, swelling, redness and pain, involved in inflammatory and immune responses. It is an inducer of other cytokines like IL-1, IL-6, IL-8, PDGF, eicosanoids, platelet activating factors and granulocyte monocyte colony stimulating factor. It exerts an interferon-like protective function against viruses, acts on the hypothalamic regulatory proteins, Inducing fever (hence called a 'pyrogen'). Also decreases appetite. 2 distinct type of receptors belonging to the TNF receptor super-family: TNF-R1 and TNF-R2. One subunit induces transcription signal and other subunit causes apoptosis.

Role in periodontitis: Direct effect on the pathogenesis of periodontal disease. P. gingivalis-induced osteoclastogenesis was reduced in TNF receptor-deficient mice compared to controls, indicating that osteoclast formation resulted from stimulation of the host response rather than from the direct effect of bacterial products.

The absence of TNFR-1 resulted in a lower production of cytokines in response to $A$. actinomycetemcomitans infection. TNF- $\alpha$ is produced mainly in response to LPS and stimulates collagenase leading to tissue destruction. It stimulates bone resorption by inducing proliferation and differentiation of osteoclast progenitors. Gaspersic et al. examined the influence of recombinant human TNF-a (rhTNF-a) on the inflammatory response and periodontal breakdown in a rat ligature model. The administration of rhTNF- $\alpha$ accelerated the progression of periodontitis in rats.

\subsubsection{Interleukin 2:}

:Produced principally by T-cells $\{$ Helper T-cells (CD4+)\}. Important for proliferation of any cell that has affinity to IL-2R like $\mathrm{T}$ and $\mathrm{B}$ lymphocytes. It is a major growth factor for $\mathrm{T}$ lymphocytes. Induces generation of $\mathrm{CD} 8+$ cytolytic $\mathrm{T}$ cells which are important is anti-viral responses. Increases effector functions of NK cells. 3 receptor forms: $\alpha, \beta, \gamma$

7.3.6 Interleukin-4

IL-4 is a pleiotropic cytokine produced by TH2 cells, mast cells and NK cells. It regulates allergic disease

7.3.7 Others interleukins

Interleukin 5: It regulates the growth, differentiation, activation and survival of eosinophils. Contributes to eosinophil migration, tissue localization and function, and blocks their apoptosis.

Interleukin 12: Regulation cytokine- initiation and regulation of cellular immune responses. Links innate and adaptive immune responses, generated from $\mathrm{T}$ and B-lymphocytes, NK cells and macrophages.

Interleukin 15: Regulates $\mathrm{T}$ cell and NK cells activation and proliferation. The number of CD8+ memory cells is shown to be controlled by a balance between IL-15 and IL-12

7.3.8 Interleukins that help in hematopoiesis

Interleukin 3: Acts as growth factor for early bone marrow progenitors. Released by activated T-lymphocytes Interleukin 7: Important mediator of the osteoclastogenesis and bone loss in inflammatory conditions such as periodontitis 
Interleukin 18: Recently discovered interleukin. Initially named as interferon $\gamma$-inducing factor, it has similar roles like IL-1 and IL-12. IL-18 is produced and released by APCs and its main targets are Th0/Th1 CD4+ T cells and NK cells

\subsection{Interferons: Anti-viral effects.}

- IFN- $\alpha$ - produced by leucocytes: Subdivided into $\alpha 1$ and $\alpha 2$

- $\quad$ IFN- $\beta$ - produced by fibroblasts

- $\quad$ IFN- $\gamma$ - produced by CD4+ cells and NK cells

Along with IL-1, it induces an increase in the expression of ICAM-1 on the cytoplasmic membrane of endothelial cells, enhancing T-lymphocyte adherence to the vascular endothelium. The principal biological actions of type I/ $\alpha$ IFNs include inhibition of viral replication, inhibition of cell proliferation, increase in the lytic potential of $\mathrm{NK}$ cells and the modulation of MHC molecule expression.

Interferon $\beta$ : It modifies the mechanics of blood barrier since it inhibits cell adhesion, cell migration and metalloproteinase activity. IFN- induces production of IL-10 and TGF, which are anti-inflammatory cytokines. IFN- $\gamma$ is an inhibitor of cell growth and proliferation.

\subsection{Chemokines}

Chemokines are a large family of chemotactic cytokines that stimulate the recruitment of inflammatory cells. Regulate the leukocyte movement. They are produced by a number of cell types in the periodontium, such as fibroblasts, endothelial cells, macrophages, osteoclasts, epithelial cells, polymorphonuclear leukocytes, monocytes, lymphocytes, and mast cells.

\subsubsection{Classification of chemokines:}

- $\quad$ CC structure $(\beta$ type)

- $\quad \mathrm{CC}$ chemokine receptor $(\mathrm{CCR})$

- $\quad$ CXC structure ( $\alpha$ type)

- $\quad$ CXC chemokine receptor (CXCR).

1. CXC/ $\alpha$ Chemokine: Alongwith IL-8 (Neutrophil- Activating factor). Released by T-lymphocytes and monocytes stimulated with TNF of IL-1. Recruits granulocytes to the area of infection

2. $\mathrm{CC} / \beta$ Chemokine: Macrophage Inflammatory proteins (MIP), RANTES (Regulated on activation, normal T-cell expressed and secreted), Macrophage Chemotactic Proteins (MCP)

\subsubsection{Biological role of chemokines:}

The primary function of chemokines is to induce the migration of leukocytes. A signal directs these cells toward the chemokines. During immunological surveillance, chemokines direct lymphocytes to the lymph nodes, which allows them to interact with the APCs and detect any invading pathogens. Some chemokines are proinflammatory in nature and require specific stimulus for their release like viral infection, bacterial products as well as other chemical agents. Proinflammatory cytokines including IL-1 and TNF- promote their release. They are nvolved in wound healing.

Role in periodontal disease: Chemokines contribute to inflammation- induced bone resorption because they can stimulate one or more steps of bone resorption, including the recruitment, differentiation, or fusion of precursor cells to form osteoclasts or enhance osteoclast survival. Chemokines also affect periodontal bone loss by their role in recruiting cells, such as neutrophils, which protect against bacterial invasion.

\section{Secretion Of Cytokines}

Antigen stimulation of TH cells in the presence of certain cytokines can lead to the generation of subpopulations of helper T cells known as Th1 and Th2. Each subset displays characteristic and different profiles of cytokine secretion. ${ }^{[10]}$

Th 1: Responsible for many cell-mediated functions (e.g., delayed-type hypersensitivity and activation of TC cells). Production of opsonization-promoting IgG antibodies. Associated with the promotion of excessive inflammation and tissue injury

Th 2: Stimulates eosinophil activation and differentiation. Provides help to B cells, and promotes the production of relatively large amounts of $\operatorname{IgM}$, IgE, and noncomplement-activating IgG isotypes. Supports allergic reactions

Key cytokines produced by each subset positively regulate the subset that produces it and negatively regulate the other subset. The progression of some diseases may depend on the balance between the TH1 and TH2 subsets. 


\section{Cytokine Storm}

A cytokine storm, also known as hypercytokinemia/ immune confusion is a potentially fatal immune reaction consisting of a positive feed back loop between cytokines and white blood cells, with highly elevated levels of various cytokines. Both pro-inflammatory cytokines (such as TNF- $\alpha$, IL- 1 , and IL-6) and antiinflammatory cytokines (such as IL-10 and IL-1Ra) are elevated in the serum of patients experiencing a cytokine storm. ${ }^{[1]}$

\section{Anti-Cytokine Therapy}

Periodontitis is an inflammatory disease fundamentally initiated by chronic bacterial infection. Microbial challenge stimulates an inflammatory host response. Host reactions to these infecting agents result in the release of inflammatory mediators including proinflammatory cytokines (IL-1, IL-6, TNF- $\alpha$ ) and prostaglandins (PGE2), which can promote extracellular matrix destruction in the gingiva and stimulate bone resorption. Although these immune and inflammatory host reactions are essential for host defense against bacterial inflammation, excessive and prolonged reaction is harmful for the functional periodontal tissue. Regulation of the immune and inflammatory reaction, in addition to controlling infection in the periodontal tissue, may be one of the methods to prevent and treat periodontal diseases.

\subsection{Indications:}

The use of the anticytokine therapy, in conjunction with anti-biofilm treatments, may prove to be advantageous.

- Patients with non-microbial risk factors, which are difficult to reduce or eliminate, such as, smoking, diabetes, and so on.

- Factors that are beyond the clinician's ability to control, such as, genetic predisposition.

- A specific group of periodontal disease susceptible individuals

\subsection{Down-regulation of cytokines:}

Cytokine receptor antagonists: Cytokine receptor antagonists bind to the receptor present on the target cell and prevent the cytokine from binding to the target cell. Therefore, there is no activation of the target cell. Example: IL-1 receptor antagonist. (IL-1Ra) Production of IL-1Ra appears to play a role in regulating the intensity of inflammatory responses. ${ }^{[12]}$

1. Soluble cytokine receptors: Soluble cytokine receptors are derived from the proteolytic cleavage of the extracellular domain of cell-bound cytokine receptors. Can be found in blood and extracellular fluid. The downregulation of cytokine is brought about by mainly two mechanisms: Soluble receptors bind to the cytokine in solution and prevent signaling. Transactivation - Soluble receptors bind the cytokine and docks on otherwise non-responsive cells and activate them.

2. Anti-cytokine antibodies: Anti-cytokine antibodies are also antagonist in function and they lower down the levels of cytokines.

\subsection{Implications on periodontal disease}

Rheumatoid arthritis is one of the best disease models suitable for anti-cytokine therapy. The principles of this strategy have been reviewed previously.There are three basic therapeutic strategies: Neutralization of cytokines, Blockage of cytokine receptors and activation of anti-inflammatory pathways such as immunosuppressive pathways

\subsection{Commercially available preparations}

Anakinra (Kineret): It is an interleukin-1 (IL-1) receptor antagonist. It competitively inhibits the binding of IL-1 to the Interleukin-1 type receptor. Anakinra blocks the biological activity of naturally occurring IL-1, including inflammation and cartilage degradation. It is used for the management of signs and symptoms of rheumatoid arthritis. The most serious side effects of Kineret are infections and neutropenia. Headache, nausea, diarrhea, flu-like symptoms and abdominal pain are also seen. 


\begin{tabular}{|c|c|c|}
\hline Agent & Nature of agent & Clinical application \\
\hline Enbrel & $\begin{array}{l}\text { Chimeric TNF-receptor/lgG } \\
\text { constant region }\end{array}$ & Rheumatoid arthritis \\
\hline Remicade & $\begin{array}{l}\text { Monoclonal antibody } \\
\text { against TNF- } \alpha \text { receptor }\end{array}$ & Rheumatoid arthritis \\
\hline Interferon $\alpha-2 a$ & Antiviral cytokine & $\begin{array}{l}\text { Hepatitis B } \\
\text { Hairy cell leukemia } \\
\text { Kaposi's sarcoma }\end{array}$ \\
\hline Interferon $\alpha-2 b$ & Antiviral cytokine & $\begin{array}{l}\text { Hepatitis C } \\
\text { Melanoma }\end{array}$ \\
\hline Interferon $\beta$ & Antiviral cytokine & Multiple sclerosis \\
\hline Actimmune & Interferon $\gamma$ & $\begin{array}{l}\text { Chronic granulomatous disease (CGD) } \\
\text { Osteopetrosis }\end{array}$ \\
\hline Neupogen & G-CSF (hematopoietic cytokine) & $\begin{array}{l}\text { Stimulates production of neutrophils } \\
\text { Reduction of infection in cancer patients } \\
\text { treated with chemotherapy }\end{array}$ \\
\hline Leukine & GM-CSF (hematopoietic cytokine) & $\begin{array}{l}\text { Stimulates production of myeloid cells after } \\
\text { bone-marrow transplantation }\end{array}$ \\
\hline Neumega & $\begin{array}{l}\text { Interleukin } 11 \text { (IL-11), a } \\
\text { hematopoietic cytokine }\end{array}$ & Stimulates production of platelets \\
\hline Epogen & $\begin{array}{l}\text { Erythopoietin (hematopoietic } \\
\text { cytokine) }\end{array}$ & Stimulates red-blood-cell production \\
\hline
\end{tabular}

11.5 Ongoing research

1. Recombinant human interleukin-11 (rhIL-11): Interleukin 11 has been shown to have anti-inflammatory effects by inhibition of TNF- $\alpha$ and other proinflammatory cytokines. IL-11 directly minimizes tissue injury through the stimulation of a tissue inhibitor of metalloproteinases-1 (TIMP-1). ${ }^{[13]}$

2. Disruption of cell signaling pathways: Strategies for preventing cell activation seek to inhibit the intracellular transduction of signals produced when ligands bind to their membrane receptors.

3. Resolvins: Resolvins stimulate the resolution of inflammation through multiple mechanisms, including preventing neutrophil penetration, phagocytosing apoptotic neutrophils to clear the lesion, and enhancing clearance of inflammation within the lesion to promote tissue regeneration. Hasturk et al. showed that, in a rabbit model of human periodontal disease, RvE1 prevents the initiation and progression of tissue destruction. ${ }^{[14]}$

\subsection{Drawbacks}

Periodontitis is an inflammatory disease fundamentally initiated by chronic infection. When inflammation is inhibited, the immune system is also downregulated. This increases the risk of microbial infection. Opportunistic infection has been reported when TNF- $\alpha$ was neutralized for rheumatoid arthritis therapy. The screening of latent infectious diseases, such as tuberculosis, should be performed before using this type of anti-cytokine therapeutic.

With antimicrobials, caution must be taken to prevent inapparent infection, without inflammatory symptoms, when anti-cytokine therapy is performed. If anti-cytokine therapy is applied to periodontal treatment, chemical plaque control reagents such as chlorhexidine gluconate in addition to mechanical control can be used.

\section{Conclusion}

Bacteria stimulate periodontal tissue destruction but that this effect is mediated by the host response induced by bacteria. By using inhibitors, it is shown that cytokines play an important role in this process. The effects of cytokines that promote osteoclast formation and bone resorption seem to be counteracted by other cytokines that are anti-inflammatory. It is probable that the balance between stimulatory and inhibitory cytokines, together with the regulation of their receptors and signaling cascades, determines the level of periodontal tissue loss. In this era of molecular biology where research has been focused on the genetic level of analysis, treatment should be focused on eliminating the root cause. Periodontal advancement should be diverted toward the use of anti-cytokine therapy in the near future. 


\section{References}

[1]. Graves DT, Delima AJ, Assuma R, Amar S, Oates T, Cochran D. IL-1 TNF antagonists inhibit the progression of inflammatory cell infiltration toward alveolar bone in experimental periodontitis. J Periodontal 1998;69:1419-25.

[2]. Jun-Ming Zhang and Jianxiong An. Cytokines, inflammation and pain. Int Anesthesiol Clin. 2007 Spring; 45(2): $27-37$.

[3]. Graves DT, Cochran D. The contribution of interleukin- 1 and tumor necrosis factor to periodontal tissue destruction. J Periodontol 2003;74:391-401.

[4]. Chiang CY, Ktritsis G, Graves DT, Amar S. IL-1 and TNF activities partially account for calvarial bone resorption induced by local injection of lipopolysaccharide. Infect Immune 1999;67:4231-6.

[5]. Graves DT, Oskoui M, Volejnikova S, et al. Tumor necrosis factor modulates fibroblast apoptosis, PMN recruitment, and osteoclast formation in response to P. gingivalis infection. J Dent Res 2001;80:1875-1879

[6]. Silva TA, Garlet GP, Fukada SY, Silva JS, Cunha FQ. Chemokines in oral inflammatory diseases: Apical periodontitis and periodontal disease. J Dent Res 2007; 86:306-319.

[7]. Murdoch C, Finn A. Chemokine receptors and the role in inflammation and infectious disease. J Am Soc Hematol 2001 95; 3032 3043.

[8]. Bannenberg GL, Chiang N, Ariel A, Arita M, Tjonahen E, Gotlinger KH, et al. Molecular circuits of resolution: Formation and actions of resolvins and protectins. J Immunol 2005;174:4345-55.

[9]. Azuma M. Fundamental mechanisms of host immune responses to infection. J Periodontal Res 2006;41: 361-373.

[10]. Van Damme J, De Ley M, Opdenakker G, Billiau A, et al. 1985. Homogenous interferon inducing 22K factor is related to endogenous pyrogen and interleukin-1. Nature. 314: 266-268.

[11]. Garlet GP, Cardoso CR, Campanelli AP, et al. The dual role of p55 tumour necrosis factor-alpha receptor in Actinobacillus actinomycetemcomitans-induced experimental periodontitis: Host protection and tissue destruction. Clin Exp Immunol 2007; 147:128-138.

[12]. Schwab JM, Chiang N, Arita M, Serhan CN. Resolvin E1 and protectin D1 activate inflammation-resolution programmes. Nature 2007;447:869-74.

[13]. Hasturk H, Kantarci A, Ohira T, Arita M, Ebrahimi N, Chiang N et al. RvE1 protects from local inflammation and osteoclastmediated bone destruction in periodontitis. FASEB J 2006;20:401-3.

[14]. Keane J, Gershon S, Wise RP, Mirabile-Levens E, Kasznica J, Schwieterman WD, et al. Tuberculosis associated with Infliximab: A TNF- $\alpha$ neutralizing agent. N Engl J Med 2001;345:1098-104. 